\title{
Learning to Predict Price based on E-commerce Online Auction Machine
}

\author{
Xiaohui $\mathrm{Li}^{\mathrm{a}, \mathrm{b}}$, Hongbin Dong ${ }^{\mathrm{a},{ }^{*}}$, Xiaowei Wang ${ }^{\mathrm{a}}$, and Shuang Han ${ }^{\mathrm{a}}$ \\ ${ }^{a}$ Computer Science and Technology College, Harbin Engineering University, Harbin, 150000, China \\ ${ }^{b}$ Harbin Vocational and Technical College, Harbin, 150000, China
}

\begin{abstract}
In this paper, we put forward a novel optimization framework entitled the E-commerce Online Auction Machine. Considering all the characteristics that affect online auction prices, the algorithms are applied to calculate the best fitting line to predict online auction prices by ordinary least squares. After that, regression weights are optimized using the local weighted method. Finally, using the shrinkage method, each characteristic optimal weight is obtained through the EOAM-RR algorithm. We have identified the key characteristics that affect auction prices as well as those that are not important.
\end{abstract}

Keywords: E-commerce online auction machine; prediction prices; regression algorithms; cross validation; online auction

(Submitted on May 12, 2018; Revised on June 19, 2018; Accepted on July 22, 2018)

(C) 2018 Totem Publisher, Inc. All rights reserved.

\section{Introduction}

With the increasing popularity of Internet technology, e-commerce and online auctions are developing rapidly. Advertisers (sellers) can display and sell commodities on e-commerce platforms, while users can shop and trade on e-commerce platforms. Many of these platforms provide several sales mechanisms for advertisers to list their items, which can be categorized into two types of listing formats: auction style listing and fixed-price style listing. E-commerce is not only one of the most important channels for transactions but also an interesting full-scale field of research. In this paper, we discuss online auctions where a seller posts a starting bid as well as a buy-it-now price.

We put forward a novel optimization framework entitled the E-commerce Online Auction Machine (EOAM), as shown in Figure 1. Figure 1 demonstrates that when a user visits an e-commerce auction website, such as a web page or a mobile app page, he can make a bid request and a bid price in the real-time auction. Generally, the winning bidder receives the auction item and pays the bid price. E-commerce Online Auction Machine considers the following three components: auction mechanism, bid price prediction, and other bid factors.

Note that our methodology aims to optimize an auction mechanism in a comprehensive optimization framework. A better auction mechanism can improve auction efficiency and profits. Thus, we directly optimize the auction time and predict bid prices. Predicting bid prices can be used to optimize the auction mechanism and improve auction efficiency.

In the E-commerce Online Auction Machine, we adopt ordinary least squares and locally weighted linear regression to predict bid prices. Moreover, we take key characteristics into consideration using ridge regression.

Using a data set collected from eBay, we construct a prediction model to predict bid prices. With the estimated model, we then conduct an analysis to present key characteristics that affect bid prices.

As a result, our contribution is comprised of the following components: (1) the major contribution is proposing the E-

\footnotetext{
* Corresponding author.

E-mail address: donghongbin@hrbeu.edu.cn
} 
commerce Online Auction Machine (EOAM), a comprehensive framework to jointly optimize an auction mechanism by predicting bid prices with bid amount and bid time constraints, (2) we point out that bid prices can be predicted by our regression modeling, and (3) experiments demonstrate how to select regression weights in eBay data sets.

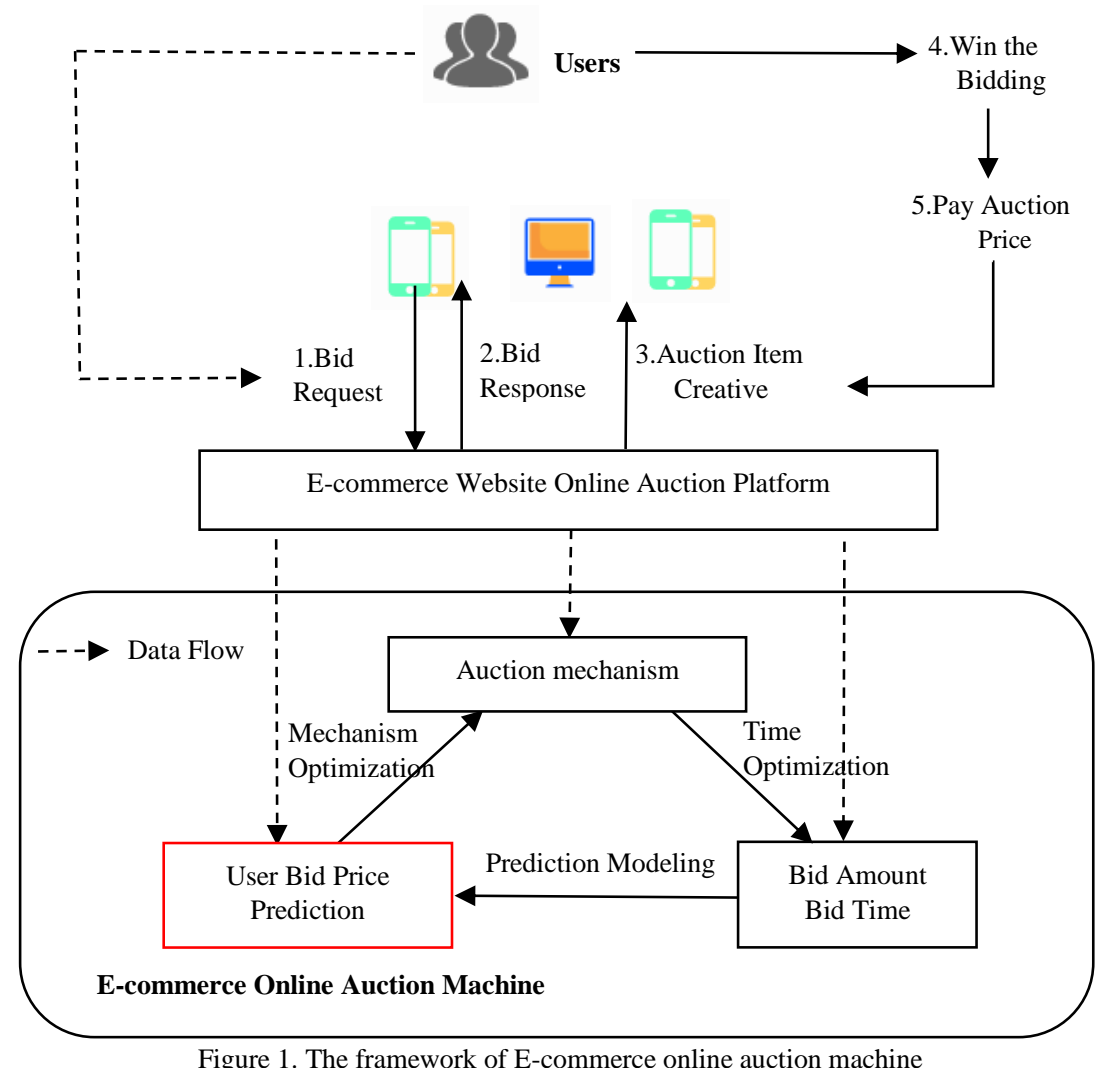

The rest of this paper is organized as follows. In Section 2, we first provide related literature and then present the advantage of our model. In Section 3, we formulate the problem and specifically introduce our proposed algorithms to calculate regression weights for predicting bid prices. Moreover, we use a real-world sample data set with eBay auctions to show the regression weights under our algorithms. After that, we analyze experimental results for the key characteristics. We further study the experiments and discuss the detailed results in Section 4. Finally, Section 5 presents the conclusions of this paper and discusses future work.

\section{Related Work}

In this section, several theoretical works about e-commerce online auctions are reviewed, and several related empirical works are also discussed. There are three main roles, including users, advertisers, and the E-commerce platform, in the framework of the E-commerce Online Auction Machine. These three roles are linked together, as all of them need to participate in a transaction. Related studies can be divided into the following categories based on the researchers' perspective. Online auctions are long in duration and offer a variety of mechanisms absent from traditional auctions. In general, there has been more research conducted on the e-commerce platform and advertisers' interests.

(i) On the aspect of E-commerce platform. In order to attract more advertisers and users, several mechanisms have been proposed. Wang [1] examines two selling mechanisms. Results indicate how to coordinate the platform's interests with those of the sellers. Acampora et al. [2] present an interval type-2 fuzzy logic based framework for reputation management in (P2P) E-commerce. The framework could protect both buyers and sellers, which is capable of better handling faced uncertainties. Vragov [3] uses experiments with economically-motivated human subjects to measure the operational efficiency of decentralized Internet auction mechanisms. Experimental results suggest that Internet auctions require a few more participants and more time to achieve operational efficiency levels than centralized markets. Selcuk [4] investigates price mechanism selection and characterizes a possible equilibrium. Greenstein-Messica [5] introduces a novel approach for a personal price aware multi-seller recommender system (PMSRS), which could improve recommendation systems' prediction accuracy. 
(ii) On the aspect of sellers. Zeithammer [6] provides auctions that are used when the seller needs to establish a separate price for every item sold. Anderson et al. [7] investigate sellers' motivation using eBay auction data sets. Sellers can choose different sale channels, so there are many works that discuss sellers' choices. Etzion et al. [8] analyze the simultaneous use of auctions and posted prices for online selling. Results suggest that the optimal design of the dual channel can significantly outperform a single posted-price channel. In general, there are a large number of sellers on an e-commerce platform, so competition among the sellers is very fierce. A handful of papers provide analyses on bidding behavior in competing auctions [9-12].

(iii) On the aspect of users (consumers). Jansen et al. [13] study users' queries on the web in real life. Jiang et al. [14] research the unique consequences of consumer behavior. B.J. Jansen et al. [15] evaluate the effectiveness of the buying funnel as a model for understanding consumer interaction with keyword advertising campaigns using the data from a major US retailer.

Unlike marketing research and result analysis, we collect data to calculate the best fitting line to predict online auction prices using our algorithms. Previously, there have been several theoretical works that conduct a relevant study to examine product characteristics. Results show that product characteristics are related to a seller's choice. Instead of only comparing the revenue, we also investigate the importance of the characteristics under the auction format (bid amount, open bid, price, duration, etc.) to present the relationship among them. Therefore, the advantage of our model could investigate the issues that have been investigated separately (the effects of open bid, price, and bid amount characteristics on format choice) in the E-commerce Online Auction Machine framework.

\section{E-commerce Online Auction Machine Algorithms}

According to the E-commerce Online Auction Machine, the optimal auction mechanism can be learned based on users' predictions of bid prices. In this section, we introduce our proposed algorithms to calculate regression weights for predicting bid prices. Algorithm 1 calculates regression weights by the minimum mean square error, and we call it EOAM-SR. For ease of reference, we summarize our algorithm in Table 1.

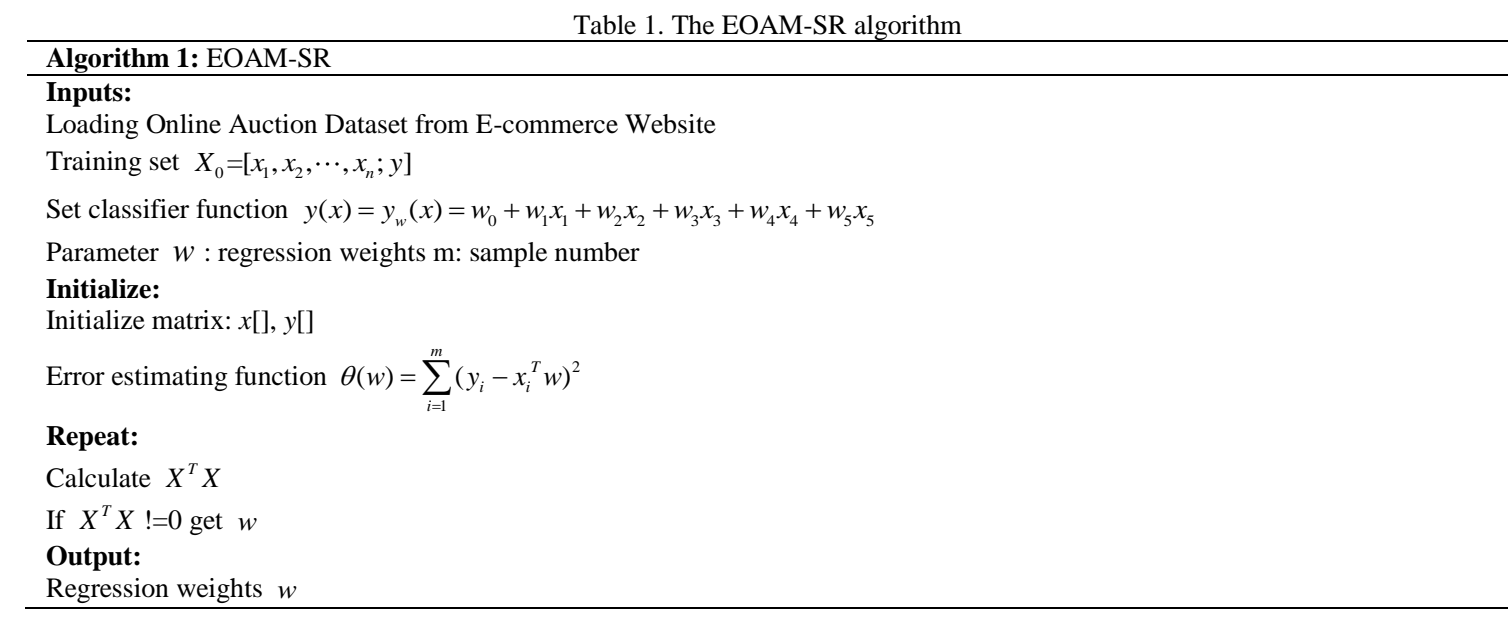

In E-commerce Online Auction Machine algorithms, the input data is stored in the matrix $X$ []. The regression weights are stored in the vector $Y[]$. The prediction results will be calculated by the linear classifier function

$$
y(x)=y_{w}(x)=w_{0}+w_{1} x_{1}+w_{2} x_{2}+w_{3} x_{3}+w_{4} x_{4}+w_{5} x_{5}
$$

Considering underfitting problems, we adopt the locally weighted algorithm. In Algorithm 2, a diagonal matrix is created first. After that, the weight is attenuated exponentially, and we call it EOAM-LWLR. For ease of reference, we summarize our algorithm in Table 2.

To prevent overfitting, we improve Algorithm 2 using the downsizing method. In Algorithm 3, the number of cross validation and data can be set, and we call it EOAM-RR. For ease of reference, we summarize our algorithm in Table 3. 
The algorithms will digest the historical bidding information and update the parameters together. The regression weights of bidding prices are also calculated in the E-commerce Online Auction Machine.

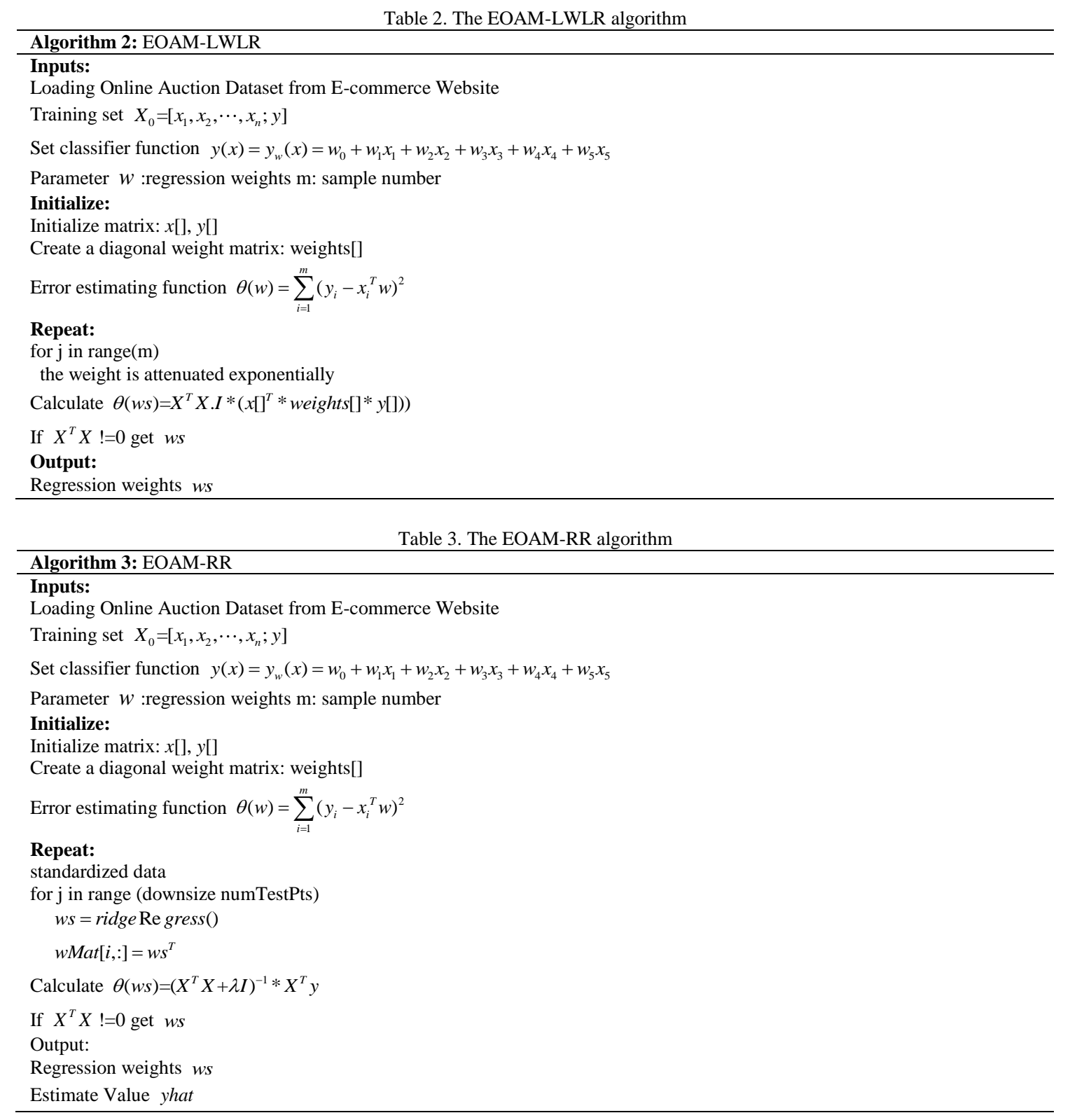

\section{Experiments}

In this section, we use a real-world sample dataset with eBay auctions on Palm Pilot PDAs. The fields used for the research reported here are shown in Table 4.

First, we present the dataset and price comparisons among auctions. Second, we present the price impact of bid characteristics. Third, we discuss the experimental results for predicting price.

Figure 2 shows the relationship between bid amount and price. We have the following observations from the figure:

- Open bid price has little effect on the final price.

- The final price increases with time.

We illustrate the impact of bid characteristics in Figure 3. The diagonal is the histogram of characteristic variables. We 
can observe the distribution of bid characteristics. The price histogram illustrates that the price obeys the normal distribution and there is hardly any abnormal value.

\begin{tabular}{|c|c|}
\multicolumn{2}{c}{ Table 4. Fields and descriptors from eBay auctions on Palm Pilot PDAs } \\
\hline Field & Description \\
\hline Auction ID & Unique identifier of an auction \\
\hline Bid amount & The proxy bid placed by a bidder \\
\hline Bid time & The time (in days) that the bid was placed, from the start of the auction \\
\hline Bidder & eBay username of the bidder \\
\hline Open bid & The opening bid set by the seller \\
\hline Price & The closing price that the item sold for (equivalent to the second highest bid + an increment) \\
\hline
\end{tabular}

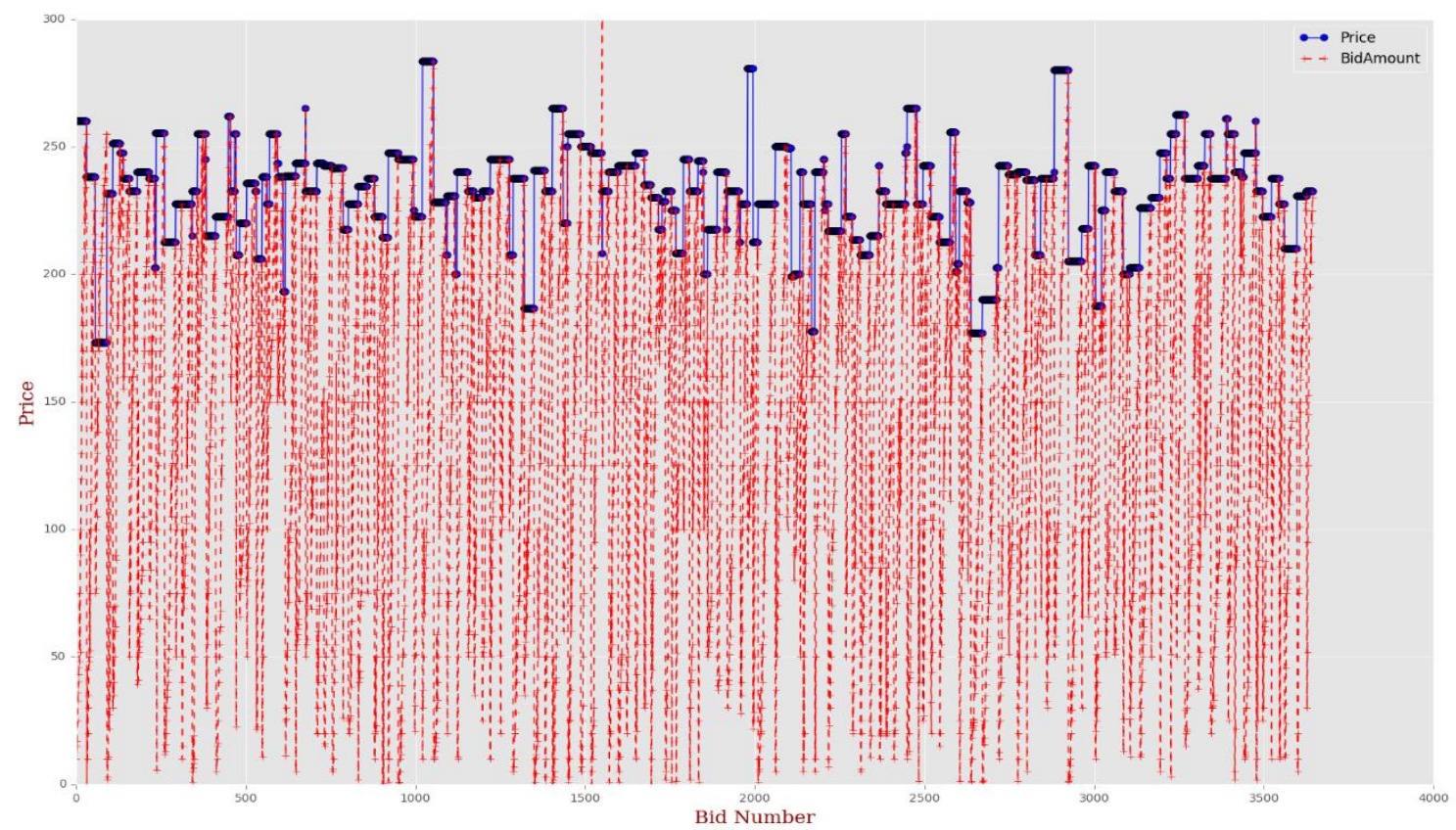

Figure 2. Price comparisons
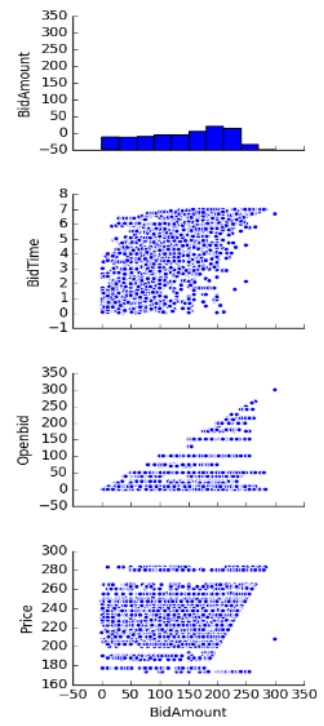
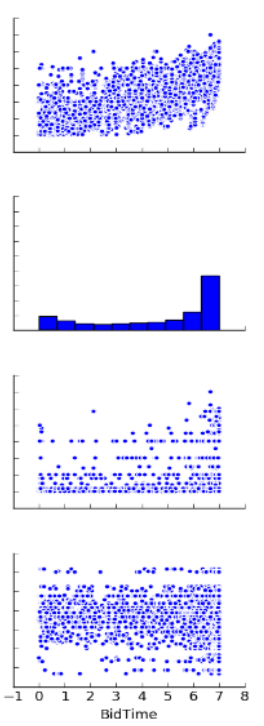
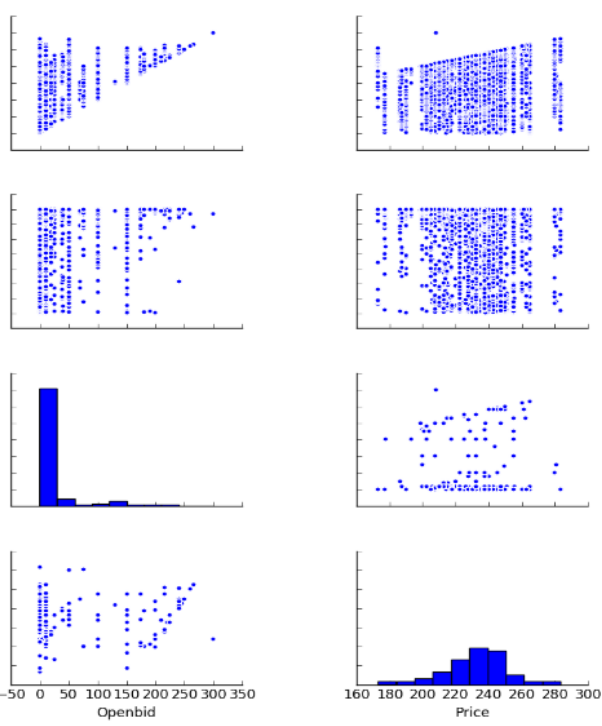

Figure 3. The illustration of the impact of bid characteristics 
To understand the impact from regression weights of Algorithm 3, we plot the bid price and predict price in Figure 4. The figure shows that the predict price is better fitted for the bid price.

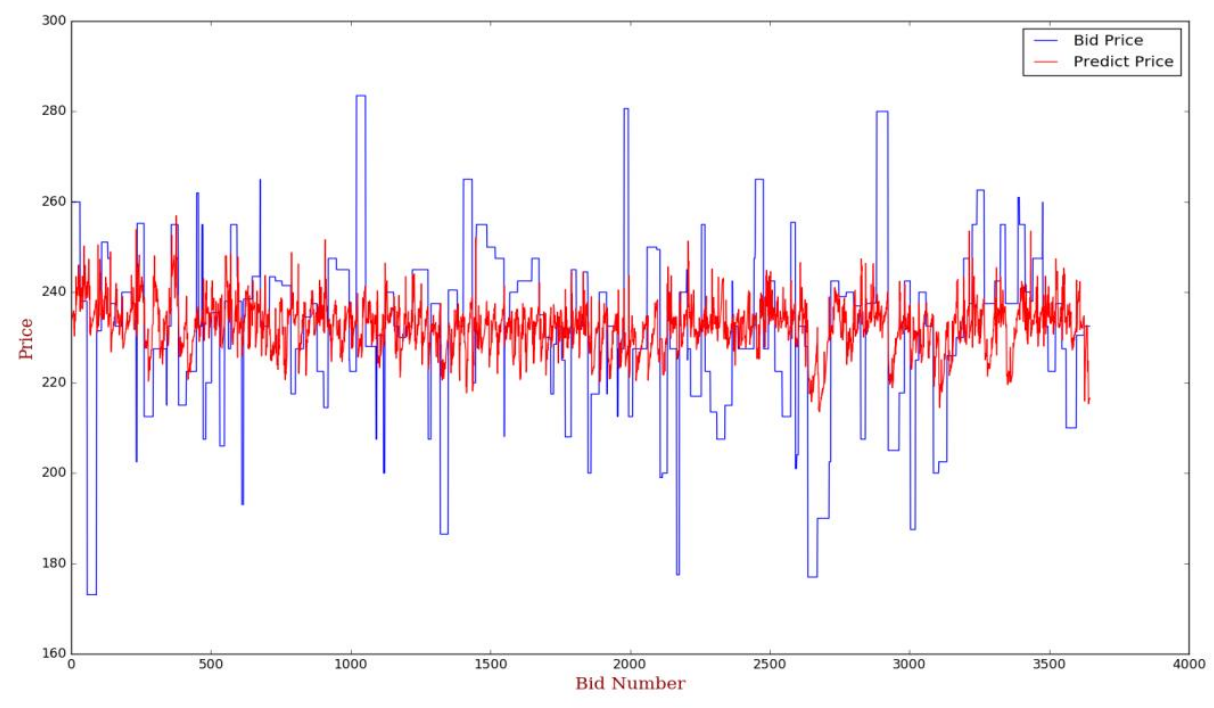

Figure 4. The relationship between bid price and predict price

Table 5 summarizes and provides a straightforward comparison of regression weights with EOAM-SR and EOAM-RR. Note that in our settings, we adopt a more reasonable validation to achieve improvement against the stand regression. Table 5 shows that $w_{1}$ is not a key characteristic and $w_{3}$ is a key characteristic.

Table 5. The result of regression weights by testing data in algorithms

\begin{tabular}{|c|c|c|c|c|c|c|}
\hline \multirow{2}{*}{ Algorithm } & \multirow{2}{*}{$\begin{array}{c}\begin{array}{c}\text { Cross validation } \\
\text { number }\end{array} \\
n\end{array}$} & \multicolumn{4}{|c|}{ Regression weights } & \multirow{2}{*}{ Constant } \\
\hline & & $w_{1}$ & $w_{2}$ & $w_{3}$ & $w_{4}$ & \\
\hline EOAM-SR & Null & $-5.11551981 e-08$ & $1.17223785 \mathrm{e}-01$ & $-3.47309458 e+00$ & $-9.49495253 e-03$ & 386.874647 \\
\hline \multirow{6}{*}{$\begin{array}{c}\text { EOAM- } \\
\text { RR }\end{array}$} & 15 & $-3.67869276 e-15$ & $-3.67869276 \mathrm{e}-15$ & $-3.27873296 e+00$ & $-7.80814860 \mathrm{e}-03$ & 232.065458987 \\
\hline & 15 & $-3.79218277 e-15$ & $1.09250893 \mathrm{e}-01$ & $-3.25677904 e+00$ & $-6.42368482 \mathrm{e}-03$ & 232.365423193 \\
\hline & 10 & $-3.53139103 e-15$ & $1.13299871 \mathrm{e}-01$ & $-3.34832344 e+00$ & $-6.40464966 \mathrm{e}-03$ & 232.223768507 \\
\hline & 10 & $-3.08276597 e-18$ & $8.99686437 \mathrm{e}-02$ & $-2.86358819 \mathrm{e}+00$ & $4.94790148 \mathrm{e}-04$ & 233.096267435 \\
\hline & 5 & $-3.87439392 e-15$ & $1.10499058 \mathrm{e}-01$ & $-3.27417903 e+00$ & $-6.71542624 \mathrm{e}-03$ & 232.276913414 \\
\hline & 5 & $-3.86180793 e-15$ & $1.14417147 \mathrm{e}-01$ & $-3.37531187 e+00$ & $-8.43422487 \mathrm{e}-03$ & 232.235031176 \\
\hline
\end{tabular}

\section{Conclusions}

E-commerce Online Auction Machine, which is a comprehensive learning system to bid and predict framework, is proposed in this paper. It aims to increase the profit and efficiency of the e-commerce platform for online auctions. Algorithms have shown how to calculate regression weights and estimate prices. This paper demonstrates our prediction model and the optimized regression weights under the e-commerce auction market. Based on the E-commerce Online Auction Machine model, we can conduct the impact of bid characteristics and predict the price under e-commerce online auctions. Selecting an eBay auction data set on Palm Pilot PDAs, we provide a regression model to investigate regression weights and analyze the key characteristics. The experiment results, including both EOAM-SR and EOAM-RR testing on an e-commercial auction platform, have the practical efficacy of our proposed E-commerce Online Auction Machine.

In the future, we aim to conduct research on improving the predicting accuracy and efficiency. We conclude with three future research directions as follows. First, we plan to integrate and test more machine learning algorithms for more accurate learning in bid landscape forecasting. Second, the extension of the E-commerce Online Auction Machine framework to reinforcement learning settings would be a promising direction. Through reinforcement learning, we could forecast and optimize commodity sorting in the framework. Finally, we hope to investigate the multi-agent bidding machine interactions and explore the potential equilibrium in the framework. 


\section{Acknowledgments}

The authors wish to gratefully acknowledge and thank the support from the National Science Foundation of China (No. 61472095).

\section{References}

1. H. Wang, “Analysis and Design for Multi-unit Online Auctions," European Journal of Operational Research, Vol. 258, No. 3, pp. 1191-1203, November 2016

2. G. Acampora, D. Alghazzawi, H. Hagras, and A. Vitiello, “An Interval Type-2 Fuzzy Logic based Framework for Reputation Management in Peer-to-Peer E-commerce," Information Sciences, Vol. 333, pp. 88-107, March 2016

3. R. Vragov, "Operational Efficiency of Decentralized Internet Auction Mechanisms," Electronic Commerce Research and Applications, Vol. 9, No. 2, pp. 111-125, March-April 2010

4. C. Selcuk, "Auctions vs. Fixed Pricing: Competing for Budget Constrained Buyers," Games and Economic Behavior, Vol. 103, pp. 262-285, May 2017

5. A. Greenstein-Messica and L. Rokach, "Personal Price Aware Multi-seller Recommender System: Evidence from eBay," Knowledge-Based Systems, Vol. 150, pp. 14-26, February 2018

6. R. Zeithammer and P. Liu, "When is Auctioning Preferred to Posting A Fixed Selling Price?" University of Chicago, November 2006

7. S. T. Anderson, D. Friedman, G. H. Milam, and N. Singh, "Buy it Now: A Hybrid Internet Market Institution," Journal of Electronic Commerce Research, Vol. 9, pp. 137-153, 2008

8. H. Etzion, E. Pinker, and A. Seidmann, "Analyzing the Simultaneous Use of Auctions and Posted Prices for Online Selling," Manufacturing \& Service Operations Management, Vol. 8, No. 1, pp. 68-91, 2006

9. R. P. Mcafee, "Mechanism Design by Competing Sellers," Econometrica, Vol. 61, No. 6, pp. 1281-1312, 1993

10. M. Peters and S. Severinov, "Internet Auctions with Many Traders," University of Toronto Working Paper, 2002

11. M. Peters and S. Severinov, "Competition among Sellers who Offer Auctions instead of Prices," Journal of Economic Theory, Vol. 75, pp. 141-197, 1997

12. A. R. Sinha and E. A. Greenleaf, "The Impact of Discrete Bidding and Bidder Aggressiveness on Sellers Strategies in Open English Auctions: Reserves and Covert Shilling,” Marketing Science, Vol. 19, No. 3, pp. 244-265, 2000

13. B. J. Jansen, A. Spink, and T. Saracevic, "Real Life, Real Users, and Real Needs: A Study and Analysis of User Queries on the Web," Information Processing \& Management, Vol. 36, No. 2, pp. 207-227, 2000

14. Y. Jiang, A. Cho, and R. Adaval, "The Unique Consequences of Feeling Lucky: Implications for Consumer Behavior," Journal of Consumer Psychology, Vol. 19, No. 2, pp. 171-184, 2009

15. B. J. Jansen and S. Schuster, "Bidding on the Buying Funnel for Sponsored Search and Keyword Advertising," Journal of Electronic Commerce Research, Vol. 12, No. 1, pp. 1-18, 2011 